\title{
High genetic diversity at the extreme range edge: nucleotide variation at nuclear loci in Scots pine (Pinus sylvestris L.) in Scotland
}

\author{
W Wachowiak ${ }^{1,2}$, MJ Salmela ${ }^{1,3}$, RA Ennos ${ }^{3}$, G Iason $^{4}$ and S Cavers ${ }^{1}$ \\ ${ }^{1}$ Centre for Ecology and Hydrology Edinburgh, Bush Estate, Penicuik, Midlothian, UK; ${ }^{2}$ Institute of Dendrology, Polish Academy

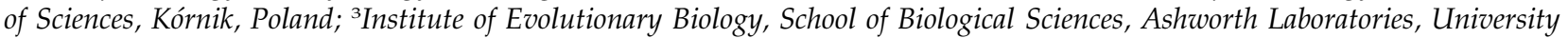 \\ of Edinburgh, Edinburgh, UK and ${ }^{4}$ Macaulay Land Use Research Institute, Craigiebuckler, Aberdeen, UK
}

\begin{abstract}
Nucleotide polymorphism at 12 nuclear loci was studied in Scots pine populations across an environmental gradient in Scotland, to evaluate the impacts of demographic history and selection on genetic diversity. At eight loci, diversity patterns were compared between Scottish and continental European populations. At these loci, a similar level of diversity $\left(\theta_{\text {sil }}=\sim 0.01\right)$ was found in Scottish vs mainland European populations, contrary to expectations for recent colonization, however, less rapid decay of linkage disequilibrium was observed in the former $(\rho=0.0086 \pm 0.0009, \rho=0.0245 \pm$ 0.0022 , respectively). Scottish populations also showed a deficit of rare nucleotide variants (multi-locus Tajima's $D=0.316$ vs $D=-0.379$ ) and differed significantly from mainland populations in allelic frequency and/or haplotype structure at several loci. Within Scotland, western
\end{abstract}

populations showed slightly reduced nucleotide diversity $\left(\pi_{\text {tot }}=0.0068\right)$ compared with those from the south and east (0.0079 and 0.0083, respectively) and about three times higher recombination to diversity ratio $(\rho / \theta=0.71$ vs 0.15 and 0.18 , respectively). By comparison with results from coalescent simulations, the observed allelic frequency spectrum in the western populations was compatible with a relatively recent bottleneck $\left(0.00175 \times 4 \mathrm{~N}_{\mathrm{e}}\right.$ generations $)$ that reduced the population to about $2 \%$ of the present size. However, heterogeneity in the allelic frequency distribution among geographical regions in Scotland suggests that subsequent admixture of populations with different demographic histories may also have played a role.

Heredity (2011) 106, 775-787; doi:10.1038/hdy.2010.118; published online 8 September 2010

Keywords: adaptation; bottleneck; nucleotide diversity; population differentiation; linkage disequilibrium; recolonization

\section{Introduction}

Nucleotide polymorphism is influenced by several factors including mutation, migration, selection and random genetic drift. In tree species, the current increase in sequence data gathered from nuclear gene loci has been driven mostly by the search for the molecular signature of natural selection (Savolainen and Pyhäjärvi, 2007; Neale and Ingvarsson, 2008; Achaz, 2009). Selection can leave its traces as deviations from neutrality in the level of nucleotide diversity, allele frequency distribution or correlation between polymorphic sites (linkage disequilibrium (LD)) (Achaz, 2009). However, the capability to detect selection at individual loci is heavily dependent on the assumptions of the neutral model (for example, constant long-term population size and random mating), the strength of, and time since, selection and the number of loci involved (and their relative effect) in selectively influenced traits (Wright and Gaut, 2005). Therefore, before testing for selection, data sets must be evaluated for violations of neutral model assumptions. Such processes, for example, historical changes in population

Correspondence: $\operatorname{Dr} S$ Cavers, Centre for Ecology and Hydrology Edinburgh, Bush Estate, Penicuik, Midlothian EH26 0QB, UK.

E-mail:scav@ceh.ac.uk

Received 4 December 2009; revised 2 July 2010; accepted 30 July 2010; published online 8 September 2010 size and distribution, may drive deviations from neutrality that mimic the effect of selection. However, these effects are expected to be genome wide and so can be distinguished from selective influences by simultaneous assessment of data from multiple loci. Although the patterns of variation in the majority of nuclear loci studied to date obey neutral expectations and the signature of selection has been elusive (Savolainen and Pyhäjärvi, 2007) polymorphisms at nuclear loci provide highly valuable insights into evolutionary history (Heuertz et al., 2006; Pyhäjärvi et al., 2007).

All northern European tree populations have experienced substantial historical changes in distribution. For example, palynological and phylogeographical data indicate that, during the last glacial maximum (2518000 years ago (ya)), most species were confined to the southern peninsulas (Iberia, Italy and the Balkans) and some parts of eastern and central Europe (Willis and van Andel, 2004; Cheddadi et al., 2006; Pyhäjärvi et al., 2008) and only reached their most northerly limits around 9000 ya. The recolonization history of forest trees, accompanied by adaptation to local environments, has potentially influenced the pattern of nucleotide diversity both among locally adapted populations and between rangeedge populations and putative refugial populations. In theory, population bottlenecks reduce nucleotide diversity in range-edge populations relative to that in source populations, although this is dependent on the 
timing and severity of the bottleneck. In contrast, admixture of populations due, for example, to recolonization from different refugia, may increase diversity (Petit et al., 2003). However, recent studies in continental European populations of Scots pine (Pyhäjärvi et al., 2007) and Norway spruce (Heuertz et al., 2006) found little evidence at the nucleotide level for the effects of recent (post-glacial) population size changes during migration and suggested bottlenecks in the mid-to-late Pleistocene. In addition, similar to other predominantly outcrossing tree species with highly efficient longdistance gene flow by pollen (Hamrick et al., 1992), neutral genetic differentiation between Scots pine populations is low. For instance, marginal population differentiation was reported for neutral markers between Finnish populations (Karhu et al., 1996), between Scandinavian and eastern parts of the range (Wang et al., 1991) and, at several candidate gene loci for growth phenology and cold tolerance, among populations along a latitudinal cline in continental Europe (Dvornyk et al., 2002; García-Gil et al., 2003). The large population sizes of forest trees and capability for maintenance of high levels of genetic variation within populations seems to further buffer against rapid changes in genetic diversity, but causes difficulties in detection of recent demographic processes. If the migrations following the most recent glaciations are to have left any signature at all in contemporary populations of forest trees, it seems likely to be detectable only where populations have experienced severe bottlenecks or became rapidly isolated.

In Scotland, Scots pine (Pinus sylvestris L.) is at the extreme north-western edge of its vast distribution, which reaches across Europe and Asia and is the largest of any pine species (Critchfield and Little, 1965). Pines first colonized the land that became the British Isles about $10000 \mathrm{ya}$, at around the time that Ireland became isolated, and reached northern Scotland by about 9000 ya (Huntley and Birks, 1983; Svendsen et al., 1999). According to fossil data in Scotland, pine first appeared in the Wester Ross region in the northwest, and then shortly afterwards in the Cairngorms in the east (Birks, 1989). The subsequent formation of the English Channel (c.6000 ya) and competition from broadleaved species in southern Britain left Scottish pinewoods physically separated by at least $500 \mathrm{~km}$ from mainland populations in continental Europe. Nowadays, native pinewoods in Scotland cover about 18000 hectares, in 84 differently sized fragments patchily distributed within a $\sim 200 \times 200 \mathrm{~km}$ area across significant environmental gradients in altitude, soil type, growing season length and annual rainfall mainly in the east-west direction (for example, annual rainfall varies from 700 to $3000 \mathrm{~mm}$ across $160 \mathrm{~km}$ ) (Mason et al., 2004). Small-scale provenance experiments have shown genetic variation between Scottish populations from different locations, for example, in root frost hardiness and growth in seedlings (Perks and McKay, 1997) and differentiation among populations at several quantitative traits (Perks and Ennos, 1999). There is reasonable evidence from pollen (Birks, 1989), allozymes, monoterpenes and mtDNA (Kinloch et al., 1986; Sinclair et al., 1998) suggesting a west/east population subdivision within Scotland and that populations from these regions may have different origins (Ballantyne and Harris, 1994; Bennett, 1995). Given the iconic status of Scots pine in
Scotland and the severe fragmentation of the population, there is considerable interest in evaluating its population history.

In this study, we focus on the Scottish Scots pine population as a unique and isolated oceanic fragment at the northwest extreme of the distribution to assess whether recent demographic processes have influenced patterns of nucleotide variation. We analyzed patterns of nucleotide diversity, allele frequency and LD in a multilocus nuclear gene data set in samples gathered from multiple locations within putatively divergent regions within Scotland and compared our data with those from samples from northern and central Europe, Turkey and Spain. Using this data and coalescent simulation analysis, we aimed to assess whether Scottish populations show the molecular signature of demographic history and the extent to which they are differentiated from those in continental Europe.

\section{Materials and methods}

\section{Sampling and DNA extraction}

Seed samples from 21 locations in Scotland were included in the study (Figure 1). The trees were sampled across an environmental gradient related to differences in altitude, length of growing season, annual rainfall and average mean temperature in winter (Supplementary Table S1). Cones were collected from mature trees in recognized old-growth Scots pine forest; at these sites trees are typically over 150 years old and are often much older (Steven and Carlisle, 1959). Trees were separated by at least $50 \mathrm{~m}$ to minimize sampling of closely related individuals. Sampling included the seven currently adopted seed zones of the species in Scotland, from each of which three locations were sampled, two individuals per location.

For most of the between-population analyses the samples were grouped according to climatic characteristics into three geographical locations-western, southern and eastern, represented by 18, 12 and 12 individuals, respectively (Figure 1, Supplementary Table S1). The western group has the lowest mean altitude ( $\sim 142 \mathrm{~m})$, the longest growing season $(\sim 240$ days), the highest mean temperature in winter $\left(\sim 2{ }^{\circ} \mathrm{C}\right)$ and high annual rainfall $(\sim 2000 \mathrm{~mm})$. The eastern group has the highest mean altitude $(\sim 372 \mathrm{~m})$, the shortest growing season ( $\sim 175$ days), and is the coldest $\left(-0.1{ }^{\circ} \mathrm{C}\right)$ and driest $(\sim 1050 \mathrm{~mm})$ part of the distribution, whereas southern group was intermediate between these extremes except for annual rainfall $(\sim 2130 \mathrm{~mm})$. Field trials have demonstrated genetic differences in phenology and growth rate among provenances originating within these groups (Perks and Ennos, 1999).

Genomic DNA was extracted from haploid megagametophyte, maternal tissue that surrounds the embryo in the seed. As DNA samples were haploid, the haplotypes could be determined by direct sequencing. In total, 42 DNA extracts were prepared, representing two different trees from each location. Seeds were germinated for a few days in moist petri dishes and then extracted following a standard CTAB (cetyltrimethylammonium bromide) protocol with addition of polyvinylpyrrolidone to $1 \%$ concentration in the lysis buffer. 


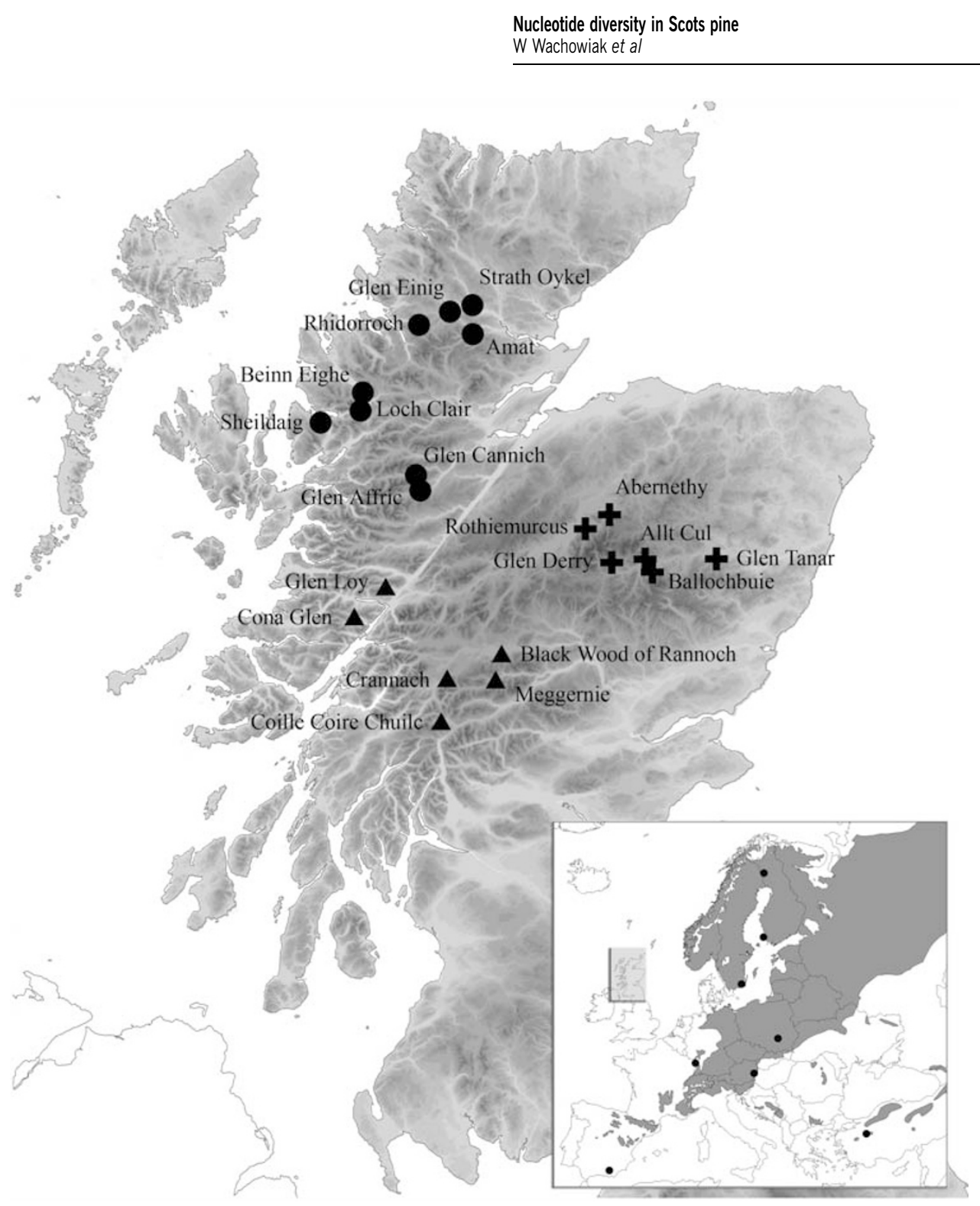

Figure 1 Main map: location of 21 Scots pine populations from Scotland (divided for most between-population analyses into groups: $\bullet$ West, $\Delta$ South and + East). Inset shows locations of the eight mainland European populations with which comparisons were made and location of main map (highlighted). See Material and methods for details.

\section{Loci studied}

In total, 16 nuclear loci were analyzed. This included several dehydrin genes that were identified in expression studies in Scots pine (Joosen et al., 2006). On the basis of the number and position of the conserved segments (Close 1997), we analyzed the class SK4 of dehydrins (dhn1), SK2 (dhn2) and a group of K2 genes (dhn3 and dhn7). We analyzed also SK type of dehydrin upregulated by water stress in Pinus taeda roots (Eveno et al., 2008) and a putative dehydrin (dhy-like) described for Scots pine (Pyhäjärvi et al., 2007). Other loci described in more detail in original papers include abscissic acid responsive protein (abaR) (Wachowiak et al., 2009); early response to dehydration 3 protein (erd3), abscissic acid, water dehydrative stress and ripening-induced gene family members 1 and 3 (lp3-1, lp3-3), Caffeoyl CoA O-methyltransferase (ccoaomt), putative arabinogalactan/proline-rich protein (PR-AGP4-1) and putative arabinogalactan/glycin-rich protein (grp3) (Eveno et al., 2008); ABI3-interacting protein 2 (a3ip2), alcohol dehydrogenase $\mathrm{C}(\mathrm{adhC})$ and chalcone synthase (chcs) (Pyhäjärvi et al., 2007).

In previous work, 10 loci (dhn1,2,3 and 7, dhy-like, dhy2PP, abaR, a3ip2, adhC and chcs) were analyzed in Scots pine from the continental European range, including 15 samples from Northern Europe (populations from Northern and Southern Finland and Sweden), 15 from Central Europe (Poland, Austria and France) and five from each of Turkey and Spain (Pyhäjärvi et al., 2007; Wachowiak et al., 2009). The reference sequences of eight loci in total (excluding dhy-like and $a d h C$, see below) were compared with those from Scottish populations. The samples from the Iberian Peninsula and Turkey were treated separately in between-region comparisons as they display specific mitochondrial types not observed in the mainland European distribution of the species that suggests different histories and no contribution to recolonization after last glaciation (Soranzo et al., 2000; Pyhäjärvi et al., 2008). 
Table 1 Loci included in nucleotide diversity analyses

\begin{tabular}{|c|c|c|c|c|c|c|c|}
\hline \multirow[t]{2}{*}{ Gene } & \multirow[t]{2}{*}{ Protein/function } & \multirow[t]{2}{*}{$\mathrm{n}$} & \multicolumn{5}{|c|}{ Base pairs screened } \\
\hline & & & Total & Coding $^{\mathrm{a}}$ & Intron & UTR & Indels ${ }^{\mathrm{b}}$ \\
\hline$d h n 1$ & Dehydrin 1-dehydrative stress response & 40 & 1265 & 489 & 423 & 353 & $10(194)$ \\
\hline$d h n 2$ & Dehydrin 2-dehydrative stress response & 33 & 449 & $235(2)$ & 119 & 95 & $2(6)$ \\
\hline dhn3 & Dehydrin 3-dehydrative stress response & 34 & 428 & $330(1)$ & - & 98 & $2(69)$ \\
\hline dhn7 & Dehydrin 7-dehydrative stress response & 38 & 364 & $264(1)$ & - & 100 & $1(2)$ \\
\hline$d h y 2 P P$ & Dehydrin-dehydrative stress response & 42 & 485 & $381(2)$ & 95 & 9 & $1(8)$ \\
\hline$a b a R$ & Abscisic acid responsive protein & 40 & 419 & $334(1)$ & 85 & - & $4(23)$ \\
\hline a3ip2 & ABI3-interacting protein 2 & 39 & 882 & $169(2)$ & 120 & 593 & $1(21)$ \\
\hline ccoaomt & Caffeoyl CoA O-methyltransferase & 41 & 563 & 316 (3) & 247 & - & 0 \\
\hline chcs & Chalcone synthase & 35 & 331 & $85(1)$ & - & 246 & $1(1)$ \\
\hline erd3 & Early responsive to dehydration 3 & 38 & 583 & 379 (3) & 204 & - & 0 \\
\hline $\operatorname{lp} 3-1$ & ABA and WDS induced gene-1 & 35 & 438 & $168(1)$ & - & 270 & $1(8)$ \\
\hline $\operatorname{lp} 3-3$ & ABA and WDS induced gene-3 & 36 & 463 & $232(2)$ & 231 & - & $6(154)$ \\
\hline Total & & 451 & 6670 & 3382 (19) & 1524 & 1764 & $29(486)$ \\
\hline
\end{tabular}

Abbreviations: $n$, haploid sample size; UTR, untranslated region.

${ }^{\mathrm{a}}$ Number of exons in parenthesis.

${ }^{b}$ Number of indels and length range in parenthesis.

\section{PCR amplification and sequencing}

PCR-amplification was performed with PTC-200 (MJ Research, Waltham, MA, USA) and carried out in a total volume of $25 \mu \mathrm{l}$ containing about $10 \mathrm{ng}$ of haploid template DNA, $50 \mu \mathrm{m}$ of each of dNTP, $0.2 \mu \mathrm{M}$ of each primer and $0.25 \mathrm{U}$ Taq DNA polymerase with the respective $1 \times$ PCR buffer (NovaZyme, Poznan, Poland). PCR followed standard amplification procedures with $\mathrm{MgCl}_{2}$ concentration optimized for each primer pair as described in Supplementary Table S2. PCR fragments were purified using QIAquick PCR Purification Kit (Qiagen, Hilden, Germany). About 20 ng of PCR product was used as a template in $10 \mu \mathrm{l}$ sequencing reactions with the Big Dye Terminator DNA Sequencing Kit (Applied Biosystems, Carlsbad, CA, USA) performed by the GenePool sequencing service, University of Edinburgh. All samples were sequenced in both directions. CodonCode Aligner software (CodonCode Corporation, Dedham, MA, USA) was used for editing and assembling of the sequence chromatograms to produce alignments on the basis of the nucleotide sequence from both DNA strands. Haplotype sequences of each locus reported in this paper are deposited in the EMBL sequence database under accession numbers GQ262040-GQ262490.

\section{Sequence analysis}

High-quality sequences were obtained for most of the samples at 12 loci (Table 1). PCR amplification or sequencing failed in most of the samples at dhy-like, $a d h C$ and grp3 and these loci, together with PR-AGP4-1 that was monomorphic across all 42 samples, were excluded from further analysis. Nucleotide sequence alignments were constructed in ClustalX and were further manually adjusted using GenDoc. All sequence polymorphisms were visually rechecked from chromatograms edited with BioEdit. Coding and non-coding regions (introns, untranslated regions) were annotated on the basis of National Center for Biotechnology Information (http://www.ncbi.nlm.nih.gov/) sequence information at each locus and web-based gene identification tool at PlantGDB (http:/ /www.plantgdb.org/cgibin/ PlantGDB/GeneSeqer/PlantGDBgs.cgi). The influence of demography on the multi-locus pattern of variation and locus-specific effects were assessed by looking at the amount of nucleotide diversity, correlation between polymorphic sites and allelic frequency distribution between different geographical locations in Scotland and in comparison to mainland populations of the species and by comparing observed statistics with simulated values under a range of demographic scenarios. Neutrality tests at intraspecific level were applied to search for departures from a neutral model of evolution. Sequences from Pinus pinaster were used as an outgroup for intraspecific comparisons to test for a signal of longerterm selection.

\section{Nucleotide diversity}

Two measures of nucleotide diversity were applied: (1) an average number of nucleotide differences per nucleotide site between two sequences $\pi$, (Nei, 1987) calculated with DNAsp 4.0, Librado and Rozas (2009) and (2) Watterson's (1975) estimate of the population mutation parameter, theta $\left(\theta_{\mathrm{W}}\right.$, equal to $4 N_{\mathrm{e}} \mu$, where $N_{\mathrm{e}}$ is the effective population size and $\mu$ is the mutation rate per nucleotide site per generation), computed on basis of the number of segregating sites and the length of each locus using MCMC simulation under a Bayesian model as previously described (Pyhäjärvi et al., 2007). The estimates of nucleotide diversity were conducted for all samples combined and separately for south, east and west regional groups of Scottish populations. Scottish and continental European populations were compared at eight loci for which informative data was available (Pyhäjärvi et al., 2007; Wachowiak et al., 2009). Exceptionally high nucleotide diversity was found at $l p 3-3$ locus compared with other loci in our data set. Owing to the size of the conifer genome and the occurrence of multi-gene families (Ahuja and Neale, 2005), erroneous co-amplification of different loci from the same family is possible and may account for unusual diversity estimates at specific loci. Therefore, locus lp3-3 was excluded from multi-locus or average estimates reported in the study to avoid bias and ensure that estimates were conservative; the locus was included in population structure analysis and coalescent simulations. 
Linkage disequilibrium and haplotype diversity

The level of LD was measured as the correlation coefficient $r^{2}$ (Hill and Robertson, 1968) using informative sites. Indels and sites with three-nucleotide variants identified in $d h n 1$ (3), dhy2PP (1) were excluded from the analysis. Under the mutation-drift-equilibrium model, the decay of LD with physical distance was estimated using non-linear regression of $r^{2}$ between polymorphic sites and the distance (in base pairs) between sites as detailed in Wachowiak et al. (2009). The nonlinear least-squares estimate of $\rho\left(\rho=4 N_{\mathrm{e}}\right.$, where $N_{\mathrm{e}}$ is effective population size, $c$ is the recombination rate) between adjacent sites was fitted by the nls-function implemented in the $R$ statistical package (http://www. r-project.org). The overall and group-specific leastsquares estimates of $\rho$ were computed and compared with other estimates in Scots pine (Pyhäjärvi et al., 2007; Wachowiak et al., 2009).

The number of haplotypes and haplotype diversity $\left(H_{\mathrm{d}}\right)$ were estimated for each gene using DNAsp. Insertions and deletions were included in all estimates. Coalescence simulations with locus-specific or average $\rho$ for six loci and without recombination were used to assess whether there are more or fewer haplotypes than expected and whether haplotype diversity is higher or lower than expected given the number of segregating sites. The number of haplotypes and haplotype diversity were calculated for all samples combined and separately for the three regional groups of Scots pine in Scotland.

\section{Neutrality tests}

Deviations of particular genes from the frequency distribution spectrum under the standard neutral model of evolution were assessed with Tajima's $D$ test (Tajima, 1989) and Fay and Wu's $H$ (Fay and Wu, 2000). Negative values of Tajima's $D$ indicate an excess of low frequency polymorphisms consistent with positive directional selection or recent population expansion, whereas positive values indicate an excess of intermediate frequency polymorphism potentially due to balancing selection or population contraction. Fay and Wu's $H$ test measures departures from neutrality on the basis of the high-frequency derived alleles. An excess of highfrequency derived alleles compared with neutral expectations may result from recent positive selection or strong population structure with uneven sampling from populations. The distribution of test statistics was investigated for each locus for all populations combined and separately for the three regional groups. Multi-locus estimates of Tajima's $D$ were assessed with HKA software (http://lifesci.rutgers.edu/ heylab). The estimates were also calculated along the sequence of each locus by a sliding window of 100 sites with successive displacement of 25 sites. As lack of recombination makes the $D$ test overly conservative (Thornton, 2005), the significance of locus-specific and multi-locus Tajima's $D$ was also evaluated by coalescent simulations dependent on population mutation and recombination rate (MANVa software www.ub.edu/softevol/manva, on the basis of the coalescent program ms, Hudson, 2002). Different estimates of $\rho$, including locus-specific estimates, lowest and highest value across loci and average value for six loci were used in coalescent simulations. As similar probability values for multi-locus $D$ statistics were observed in simulations with different recombina- tion rate estimates, the results based on the average values of $\rho$ at the analyzed loci are reported unless otherwise stated.

For tests based on nucleotide variation between species we used reference sequence data from $P$. pinaster for outgroup comparison. To assess the correlation between the level of nucleotide polymorphism and divergence at each locus we applied (1) the McDonald and Kreitman (1991) test, on the basis of comparison of the pattern of within-species polymorphism and between-species divergence at synonymous and nonsynonymous sites in a gene and (2) HKA test (Hudson et al., 1987), which allows the detection of loci that demonstrate unusual patterns of polymorphism compared with divergence across genes. Comparison of multi-locus polymorphism and divergence at all sites was assessed using HKA software (http:/ / lifesci.rutgers. $\mathrm{edu} / \sim$ heylab). The ratio of non-synonymous $(\mathrm{Ka})$ and synonymous site $(K s)$ nucleotide divergence from the outgroup species (Hughes and Nei, 1988) was calculated using DNAsp.

\section{Population structure}

To check whether there was a geographical difference in allelic frequency spectra, regional groups of Scottish populations were compared with each other and to previously analyzed continental European populations from northern and central Europe, Spain and Turkey (Pyhäjärvi et al., 2007; Wachowiak et al., 2009). Genetic differentiation between the regions was studied locus by locus at both haplotype and single-nucleotide polymorphism/Indel level and also by averaging pairwise $F_{\mathrm{ST}}$ over all polymorphic sites across loci. The significance of genetic differentiation was evaluated by 1000 permutations of the samples between groups using Arlequin ver. 3.0 (Excoffier et al., 2005). Population structure from the haplotypic data was tested by $S_{\mathrm{nn}}$ and $K_{\mathrm{ST}}^{*}$ statistics (Lynch and Crease, 1990; Hudson et al., 1992), which are more appropriate for sequence-based haplotype data where diversity may be high and sample size low, rendering frequency-based approaches problematic. Their significance was evaluated using 1000 permutations, where samples were randomly assigned into different groups (Hudson, 2000). Genetic clustering of the individuals on the basis of both full sequence data and all segregating sites and indels at 12 loci (for Scottish populations) and at 8 loci (for Scottish and mainland European populations) was conducted using BAPS 5.2 (Corander and Tang, 2007). Polymorphic sites from each locus were treated as linked molecular data to account for dependence between segregating sites in the gene. Completely linked sites $\left(r^{2}=1\right)$ were excluded from the analysis.

\section{Coalescent simulations}

To further infer the demographic history of Scottish Scots pine populations we compared the observed distribution of average Tajima's $D$ and Fay and Wu's $H$ at the candidate loci separately in the western, southern and eastern group and in all geographical regions combined to the simulated values under several demographic scenarios, including the standard neutral model (constant population size), growth model and bottleneck model followed by exponential growth (Supplementary Figure S4). Regional groups of populations were 
Table 2 Summary statistics of nucleotide and haplotype variation and frequency distribution spectrum of polymorphism at analyzed genes in Scottish populations of Scots pine

\begin{tabular}{|c|c|c|c|c|c|c|c|c|c|c|c|c|c|c|}
\hline \multirow[t]{3}{*}{ Locus } & \multirow[t]{3}{*}{$\mathrm{L}$} & \multicolumn{8}{|c|}{ Nucleotide diversity } & \multirow[t]{3}{*}{$\rho^{\mathrm{b}}(S E)$} & \multirow[t]{3}{*}{$D^{\mathrm{c}}$} & \multirow[t]{3}{*}{$H^{\mathrm{d}}$} & \multicolumn{2}{|c|}{ Haplotype diversity } \\
\hline & & \multirow[b]{2}{*}{$\pi$} & \multicolumn{2}{|c|}{ Non-synonymous } & \multicolumn{5}{|c|}{ Silent ${ }^{\mathrm{a}}$} & & & & \multirow[t]{2}{*}{$N$} & \multirow[t]{2}{*}{$H_{d}(S D)$} \\
\hline & & & SNPS & $\pi$ & SNP & $\pi$ & $\pi_{\text {West }}$ & $\pi_{\text {South }}$ & $\pi_{\text {East }}$ & & & & & \\
\hline$d h n 1$ & 1071 & 0.0144 & $8(4)$ & 0.0044 & $54(12)$ & 0.0203 & 0.0215 & 0.0225 & 0.0158 & $0.0113(0.0012)$ & 0.039 & -0.285 & 24 & $0.964(0.014)$ \\
\hline$d h n 2$ & 442 & 0.0074 & 1 & 0.0024 & $7(0)$ & 0.0112 & 0.0107 & 0.0110 & 0.0113 & $0.0779(0.0379)$ & $1.968^{1,2, *}$ & 0.458 & 11 & $0.888(0.028)$ \\
\hline dhn3 & 359 & 0.0198 & $10(2)$ & 0.0137 & $13(2)$ & 0.0291 & 0.0325 & 0.0226 & 0.0366 & - & 0.911 & 1.733 & 8 & $0.829(0.03)$ \\
\hline$d h n 7$ & 362 & 0.0042 & $3(1)$ & 0.0042 & $4(3)$ & 0.0043 & 0.0033 & 0.0036 & 0.0061 & - & -0.223 & -2.339 & 6 & $0.711(0.039)$ \\
\hline dhy $2 P P$ & 476 & 0.0101 & $5(3)$ & 0.0011 & $12(2)$ & 0.0244 & 0.0240 & 0.0223 & 0.0257 & $0.0680(0.0228)$ & 0.103 & 1.738 & 21 & $0.954(0.015)$ \\
\hline$a b a R$ & 396 & 0.0048 & $5(0)$ & 0.0052 & $3(2)$ & 0.0043 & 0.0035 & 0.0048 & 0.0046 & - & 0.052 & -2.323 & 10 & $0.755(0.057)$ \\
\hline a3iP2 & 861 & 0.0043 & $2(2)$ & 0.0008 & $12(5)$ & 0.0049 & 0.0051 & 0.0045 & 0.0054 & $0.0022(0.0016)$ & 0.360 & -2.231 & 11 & $0.779(0.052)$ \\
\hline ccoaomt & 563 & 0.0018 & $1(1)$ & 0.0000 & $5(0)$ & 0.0032 & 0.0011 & 0.0053 & 0.0038 & - & -0.711 & -0.746 & 5 & $0.348(0.092)$ \\
\hline chcs & 330 & 0.0075 & $1(1)$ & 0.0008 & $12(6)$ & 0.0094 & 0.0066 & 0.0137 & 0.0088 & - & -0.682 & -1.267 & 9 & $0.766(0.065)$ \\
\hline erd3 & 583 & 0.0021 & $3(3)$ & 0.0006 & $7(4)$ & 0.0037 & 0.0036 & 0.0029 & 0.0046 & - & $-1.464^{2, *}$ & -1.351 & 9 & $0.73(0.052)$ \\
\hline lp3-1 & 430 & 0.0095 & $1(1)$ & 0.0004 & $12(1)$ & 0.0137 & 0.0107 & 0.0144 & 0.0135 & $0.0195(0.0111)$ & 0.944 & 1.175 & 20 & $0.955(0.017)$ \\
\hline lp3-3 & 309 & 0.0370 & $8(0)$ & 0.0177 & $18(1)$ & 0.0660 & 0.0692 & 0.0683 & 0.0676 & $0.0018(0.0011)$ & $2.846^{1,2, * * *}$ & 1.600 & 19 & $0.949(0.019)$ \\
\hline Total & 6182 & & $48(18)$ & & $159(38)$ & & & & & & & & & \\
\hline Mean $^{\mathrm{e}}$ & 515 & 0.0078 & & 0.0031 & & 0.0117 & 0.0111 & 0.0116 & 0.0124 & $0.0085(0.0009)^{\mathrm{f}}$ & 0.118 & -0.494 & 12.18 & $0.789(0.042)$ \\
\hline
\end{tabular}

Abbreviations: L, length of sequence in base pairs excluding indels; $H_{\mathrm{d}}$, haplotype diversity (standard deviation); $N$-number of haplotypes; SE, standard error; SNP, single-nucleotide polymorphism.

Silent sites variation reported separately for west, south and east geographical groups, otherwise average values for all samples combined. $\pi$-nucleotide diversity (Nei, 1987).

asynonymous and non-coding positions.

${ }^{\mathrm{b}}$ Least-squares estimate of recombination parameter.

${ }^{c} D$ test (Tajima, 1989).

${ }^{\mathrm{d}} \mathrm{H}$ test (Fay and $\left.\mathrm{Wu}, 2000\right)$.

eAverage values at 11 loci excluding $l p 3-3$.

fEstimates based on informative sites at all loci, excluding $l p 3-3$; '- ' not estimated because of the low number of informative sites.

*Statistically significant values based on coalescence simulations (1) without recombination and (2) with average recombination rate at six loci ${ }^{*} P<0.05 ;{ }^{* * *} P<0.01$.

analyzed separately as detailed aspects of the frequency spectrum may differ between groups that are not differentiated on the basis of the genetic clustering methods (Pyhäjärvi et al., 2007). Coalescent simulations were run independently for each locus and various demographic scenarios using the program ms (Hudson 2002) and the approach described by Haddrill et al. (2005). In each case, 5000 replicates were simulated for each locus. The analyses were performed with recombination using the locus-specific (when available) or average value of $\rho$ per site for the analyzed loci in each geographical group (Table 2, Supplementary Table S3). We tested various bottleneck scenarios of different age and severity. The time from the end of the bottleneck (measured in units of $4 N_{0}$ generations from the present) ranged from 0.0002 to 0.05 and bottleneck severities (measured in units of the current population size) from 0.001 to 0.5 . Assuming for instance, $N_{\mathrm{e}}$ of 200000 and generation time of 25 years, the time range corresponds to between 4000 and 1 million years and severity from 0.1 to $50 \%$ of the current population size. In most bottleneck models tested, the ancestral and current effective population sizes were assumed to be equal, bottleneck duration $(f)$ was fixed to $f=0.0015$ (units of $4 N_{0}$ generations from the present) and the growth rate of 10 was constant across simulations as in previous studies (Heuertz et al., 2006). A subset of simulations were also run with $f=0.006$ and corresponding equal or doubled ancestral population size as compared with the current one, and also separately for a set of 11 and 9 loci (excluding lp3-3 and $d h n 1$ and $a b a R$, respectively as the later showed some evidence of selection). A schematic representation of the simulated bottleneck model is shown in Supplementary Figure S4. The simulation results for each demographic scenario were summarized using the program analyser HKA. The perl script multitest_pop1.pl was used to perform multi-locus tests of ms-generated genealogies (including $P$-values of the observed mean values of Tajima's $D$ and Fay and Wu's $H$ statistics) summarized using analyser HKA. The programs are available from http://genomics.princeton.edu/AndolfattoLab/ Andolfatto_Lab.html.

\section{Results}

\section{Nucleotide polymorphism and divergence}

The average total nucleotide diversity $(\pi)$ in Scottish populations at 11 loci was $\pi_{\text {tot }}=0.0078$ and at nonsynonymous sites was $\pi_{\mathrm{ns}}=0.0031$ (Table 2). Slightly lower average nucleotide diversity was found in the west $\left(\pi_{\text {tot }}=0.0068\right)$ as compared with southern and eastern regional groups $\left(\pi_{\text {tot }}=0.0079\right.$ and 0.0083 , respectively) and similar values were found at non-synonymous sites $\left(\pi_{\mathrm{ns}}=\sim 0.003\right) \quad$ (Supplementary Table S3). Multi-locus estimates of silent Watterson theta was $\theta_{\text {sil }}=0.0095$ (with 95\% credibility intervals of $0.0074-0.0122$ ) for all Scottish populations combined, $\theta_{\text {sil }}=0.0086(0.0063-0.0117)$ in the west, $\theta_{\text {sil }}=0.0111(0.0080-0.0152)$ in the south and $\theta_{\text {sil }}=0.0103(0.0074-0.0143)$ in the east. In comparisons between Scottish vs mainland European populations at eight loci, similar but slightly higher average values of total nucleotide diversity $\left(\pi_{\text {tot }}=0.0070\right.$ vs 0.0062$)$ and silent multi-locus theta $\left(\theta_{\mathrm{sil}}=0.0108\right.$ vs 0.0093$)$ were found in Scottish populations (Table 3). 
Table 3 Descriptive statistics for nucleotide variation at eight loci in Scottish and continental European populations of Scots pine. Description of regional groups in Scotland as in Figure 1 and Supplementary Table S1

\begin{tabular}{|c|c|c|c|c|c|c|}
\hline Groups & $\theta^{\mathrm{a}}$ & $C I(95 \%)^{\mathrm{b}}$ & $\rho(S E)^{\mathrm{c}}$ & $\rho / \theta$ & $D^{\mathrm{d}}$ & $H^{\mathrm{e}}$ \\
\hline \multicolumn{7}{|l|}{ Scottish } \\
\hline West & 0.0103 & $0.0072-0.0147$ & $0.0073(0.0008)$ & 0.71 & $0.580^{*}$ & -0.400 \\
\hline South & 0.0130 & $0.0089-0.0188$ & $0.0020(0.0004)$ & 0.15 & 0.107 & 0.066 \\
\hline East & 0.0117 & $0.0080-0.0170$ & $0.0021(0.0006)$ & 0.18 & $0.499 *$ & -0.128 \\
\hline All & 0.0108 & $0.0081-0.0145$ & $0.0085(0.0009)$ & 0.79 & 0.316 & -0.564 \\
\hline \multicolumn{7}{|c|}{ Continental European } \\
\hline North $^{\mathrm{f}}$ & 0.0095 & $0.0065-0.0137$ & $0.0062(0.0010)$ & 0.65 & -0.143 & -0.750 \\
\hline Central $^{g}$ & 0.0103 & $0.0072-0.0147$ & $0.0090(0.0009)$ & 0.87 & -0.359 & -1.077 \\
\hline North+Central & 0.0096 & $0.0070-0.0131$ & $0.0214(0.0019)$ & 0.45 & -0.316 & -1.116 \\
\hline Spain & 0.0098 & $0.0058-0.0167$ & - & - & -0.539 & -0.371 \\
\hline Turkey & 0.0055 & $0.0030-0.0099$ & - & - & -0.279 & -0.792 \\
\hline All & 0.0093 & $0.0068-0.0125$ & $0.0245(0.0022)$ & 2.69 & -0.379 & -1.240 \\
\hline
\end{tabular}

Abbreviations: CI, credibility interval; SE, standard error.

${ }^{a}$ Median for silent sites.

$\mathrm{b}_{95} \%$ credibility intervals for $\theta$.

${ }^{c}$ Least-squares estimate of $\rho$.

d'Tajima's $D$ test based on all sites.

${ }^{\mathrm{e}}$ Fay and $\mathrm{Wu} H$ test.

${ }^{f}$ North: Finland North, Finland South and Sweden.

gCentral: Poland, France and Austria; '- ' not estimated because of the low sample size ( $~ 5$ for each locus) and low number of informative sites from each population.

${ }^{*} P<0.05$, statistical significance determined by coalescent simulations with and without recombination (see Materials and methods).

\section{Linkage disequilibrium and haplotype polymorphisms}

Rapid decay of LD between pairs of parsimony informative sites at 11 loci was found in Scottish populations, with $\rho=0.0085 \pm 0.0009$ (Table 2) and expected $r^{2}$ values of 0.2 at a distance of about $400 \mathrm{bp}$. The decay of LD in the western group ( $\rho=0.0074 \pm 0.0008$ ) was more rapid as compared with the south $(0.0025 \pm$ $0.0004)$ and east $(0.0024 \pm 0.0006)$ (Figure 2) and the pattern was constant at most loci (Supplementary Table S3). Overall, Scottish populations had about three times slower decay of LD as compared with mainland populations at the same set of eight loci of similar sample size ( $\rho=0.0085 \pm 0.0009$ vs $0.0245 \pm 0.0022$, respectively) (Supplementary Figure S1). However, the rate of decay of $\mathrm{LD}$ and the relative level of recombination to diversity $(\rho / \theta$ ratio) were similar between western Scottish and north and central European regions (Table 3), but these parameters were over three times smaller in southern and eastern groups of Scotland.

The average number of haplotypes per gene was 12 and haplotype diversity was very high $\left(H_{\mathrm{d}}=0.789 \pm\right.$ $0.042)$. Similar haplotype diversity was found in western $\left(H_{\mathrm{d}}=0.754 \pm 0.077\right)$, southern $\left(H_{\mathrm{d}}=0.819 \pm 0.088\right)$ and eastern $\left(H_{\mathrm{d}}=0.800 \pm 0.090\right)$ groups (Supplementary Table S3). Haplotype diversity was slightly higher than mainland European populations at the same set of eight loci $\left(H_{\mathrm{d}}=0.831 \pm 0.038\right.$ vs $\left.H_{\mathrm{d}}=0.795 \pm 0.051\right)$ and also compared with previous estimates for Scots pine $\left(H_{\mathrm{d}}=0.683 \pm 0.059\right.$, Wachowiak et al., 2009). Locus Lp3-3 contained two sets of haplotypes (each of 18 samples equally distributed across three geographical groups) with highly reduced levels of nucleotide polymorphism $\left(\pi_{\text {tot }}=0.0090\right.$ and 0.0074 , respectively) as compared with the whole gene estimate $\left(\pi_{\text {tot }}=0.0370\right)$ and a 10 -fold difference in the level of divergence $\left(K_{\text {sil } 1}=0.013\right.$ vs $K_{\text {sil2 }}=0.116$ ) (Supplementary Table S4 and Supplementary Figure S2). A neutral coalescence process, compatible with a constant-size neutral model without recombination or erroneous co-amplifications of different gene family members could potentially generate such a pattern. However, no reading-frame shifts or premature stop codons, which would suggest the presence of nonfunctional alleles, were found at the locus.

\section{Neutrality tests}

Tendency towards an excess of old over recent mutations across genes was detected by multi-locus Tajima's $D$ at 11 loci in the total data set $(D=0.118)$ (Table 2), in the western $(D=0.364)$, southern $(D=0.103)$ and eastern $(D=0.260)$ groups (Supplementary Table S3). Significant excess of intermediate frequency mutations was found at $d h n 2$ $(D=1.968, P<0.05)$ and $\operatorname{lp} 3-3(D=2.846, P<0.01)$. Statistically significant positive values of Tajima's $D$ were identified in sliding window analyses in a few regions within dhn2 ( $D=2.36-2.48$ at 307-449 bp), a3ip2 $(D=2.22$ at $401-501 \mathrm{bp})$ and $\operatorname{lp} 3-3(D=2.13-3.18$ at $51-454 \mathrm{bp})$ loci. Overall, an excess of high-frequency derived variants indicated by negative mean values of Fay and Wu's $H$ statistics was found in all Scottish populations $(H=-0.494)$ (Table 2), in the west $(H=-0.447)$ and east $(H=-0.145)$ groups, but slightly positive values were found in the south $(H=0.144)$ (Supplementary Table S3). The aggregated Scottish populations show a deficit of rare variants (multi-locus Tajima's $D=0.316$ ) as compared with mainland European populations $(D=-0.379)$. Both geographical regions show negative mean values of Fay and Wu's $H$ statistic $(H=-0.564$ and -1.240 , respectively) indicating an excess of high-frequency derived single-nucleotide polymorphysms (Table 3).

An excess of fixed non-synonymous over fixed synonymous substitutions and polymorphic sites was found at $d h n 1$ locus in McDonald-Kreitman test (Fisher's exact test, $P=0.05$ ), as previously found in European 

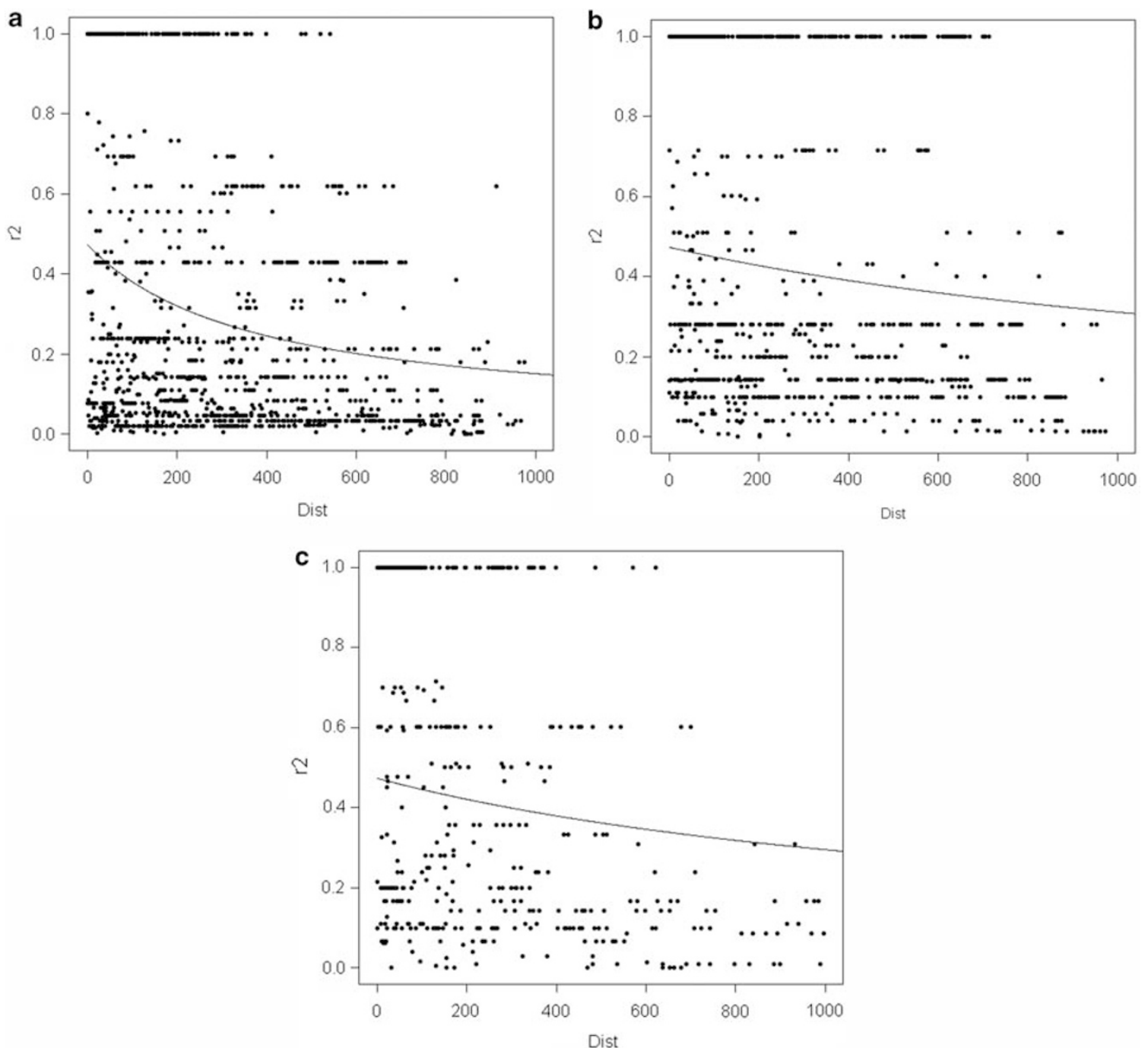

Figure 2 Scatter plots of the squared correlation coefficient of allele frequencies $\left(r^{2}\right)$ as a function of distance in base pairs between pairs of polymorphic sites in western (a), southern (b) and eastern (c) groups at all loci combined. Decline in linkage disequilibrium is shown by non-linear fitting curve of the mutation-recombination-drift model (see Material and methods section for details). Recombination rate parameter $\rho$ (standard error in parenthesis) for western group is $\rho=0.0074(0.0008)$, for southern group is $\rho=0.0025(0.0004)$ and $\rho=0.0024(0.0006)$ for the east.

mainland populations (Wachowiak et al., 2009). An excess of non-synonymous sites as compared with synonymous sites was found at $a b a R$ (Supplementary Table S5). The level of divergence was similar across all sites and at silent sites only $(\sim 4 \%)$, and was slightly lower than previous estimates for Scots pine ( $K=\sim 0.05$, Wachowiak et al., 2009). Overall, positive correlation between polymorphism and divergence (HKA test) was found at 11 loci combined.

\section{Population differentiation}

Differentiation between Scottish populations: Significant differentiation measured as an average over all polymorphic sites was found between southern and eastern groups at dhn1 $\left(F_{\mathrm{ST}}=0.034, P<0.05\right)$ and between southern and eastern as compared with the western group at ccoaomt $\left(F_{\mathrm{ST}}=0.149, P<0.05\right.$ and $F_{\mathrm{ST}}=0.102$, $P<0.01$, respectively $)$ and $l p 3-1\left(F_{\mathrm{ST}}=0.100, P<0.05\right.$ and $F_{\mathrm{ST}}=0.197, P<0.001$, respectively) (Supplementary Table S6). A difference in frequency of indel polymorphisms at $d h n 1$, four silent substitutions and indel polymorphisms at $l p 3-1$ and absence of four silent polymorphisms in the western group as compared with the others at ccoaomt locus contributed the most to the differentiation between groups. On the basis of the haplotype differentiation, the western group differed from the southern group at a3ip2 $\left(S_{\mathrm{nn}}=0.629, P<0.05\right), \operatorname{lp} 3-1\left(S_{\mathrm{nn}}=0.758, P<0.01\right)$ and $\mathrm{at}$ ccoaomt and lp3-1 on the basis of the $K_{\mathrm{ST}}$ statistics $\left(K_{\mathrm{ST}}=0.066\right.$ and $0.051, P<0.05$, respectively). They also differ from the eastern group at $l p 3-1$ locus $\left(K_{\mathrm{ST}}=0.075\right.$, $P \ll 0.05)$. Significant $F_{\mathrm{ST}}$ statistics based on haplotype frequency were found for $1 p 3-1$ in the south and east as compared with western group $(P<0.05)$ and nearly significant values for ccoaomt between south and west groups $(P=0.06)$ (Supplementary Table S6). No difference between west-south, west-east and south-east groups were found on the basis of the average $F_{\mathrm{ST}}$ over all polymorphic sites and indels combined across the loci $\left(F_{\mathrm{ST}}=-0.013,-0.013\right.$ and 0.01 , respectively $)$.

Differentiation between Scottish vs European continental populations: On the basis of the allele frequency and/or haplotype diversity statistics Scottish populations were 
Table 4 Differentiation between Scottish and continental European populations of Scots pine measured as average $F_{\mathrm{ST}}$ over all polymorphic sites and indels at eight loci combined

\begin{tabular}{|c|c|c|c|c|c|c|}
\hline & North & Central & Spain & Turkey & North + Central & $A l l^{\mathrm{a}}$ \\
\hline West & $0.032^{* * *}$ & $0.026^{* *}$ & 0.02 & $0.091^{* * *}$ & $0.029^{*}$ & $0.022^{*}$ \\
\hline South & 0.009 & 0.011 & $0.053^{* *}$ & $0.112^{* * *}$ & 0.010 & 0.011 \\
\hline East & 0.019 & $0.040^{* * *}$ & $0.072^{* *}$ & $0.145^{* * *}$ & $0.037^{*}$ & $0.039^{*}$ \\
\hline All Scottish & $0.023^{* *}$ & $0.035^{* *}$ & $0.035^{*}$ & $0.095^{* * *}$ & $0.028^{*}$ & $0.025^{*}$ \\
\hline
\end{tabular}

${ }^{a}$ All continental European populations combined; ${ }^{*} P<0.05,{ }^{* *} P<0.01$ and ${ }^{* * *} P<0.001$.

Table 5 Alternative demographic models tested against total and regional groups of populations in Scotland

\begin{tabular}{|c|c|c|c|c|c|c|c|c|}
\hline \multirow[t]{2}{*}{ Group } & \multicolumn{2}{|c|}{ Observed $^{\mathrm{a}}$} & \multicolumn{2}{|c|}{ SNM } & \multicolumn{2}{|c|}{ Growth $^{\mathrm{b}}$} & \multicolumn{2}{|c|}{ Bottleneck } \\
\hline & Mean D & Mean $H$ & Mean D & Mean $H$ & Mean $D$ & Mean $H$ & Mean $D$ & Mean $H$ \\
\hline West & 0.364 & -0.447 & $-0.057(0.578)$ & $0.001(0.406)$ & $-0.059(0.585)$ & $-0.018(0.411)$ & $0.371(0.116)$ & $-0.504(0.630)$ \\
\hline South & 0.103 & 0.144 & $-0.066(0.588)$ & $-0.009(0.419)$ & $-0.056(0.575)$ & $0.023(0.403)$ & $0.310(0.161)$ & $-0.494(0.636)$ \\
\hline East & 0.260 & -0.145 & $-0.056(0.589)$ & $0.003(0.419)$ & $-0.065(0.596)$ & $-0.013(0.434)$ & $0.311(0.165)$ & $-0.487(0.645)$ \\
\hline All & -0.015 & -0.494 & $-0.072(0.602)$ & $0.028(0.407)$ & $-0.072(0.605)$ & $0.026(0.406)$ & $0.661(0.020)$ & $-0.495(0.613)$ \\
\hline
\end{tabular}

Abbreviation: SNM, Standard neutral model.

a'Observed mean values of Tajima's $D$ and Fay and Wu's $H$ statistics at 11 loci.

${ }^{\mathrm{b}}$ Results for exponential growth of rate 10 starting $0.00125 \times 4 N_{\mathrm{e}}$ before present.

${ }^{c}$ Results shown are for bottleneck of severity $s=0.02$ that started $0.00175 \times 4 N_{\mathrm{e}}$ generations before present. Duration of bottleneck was set up to 0.0015 and population growth rate to 10 . Assuming e.g. $N_{\mathrm{e}}=200000$ and generation time of 25 years, the bottleneck ended about 25 thousand years ago. Current and the ancestral population size were assumed to be equal. In parenthesis are the $P$-values for the observed means of each parameter.

differentiated from continental European populations at six out of eight loci analyzed (Supplementary Table S7). Significant population differentiation $\left(F_{\mathrm{ST}}\right)$, measured both as an average over polymorphic sites and at the haplotype level, was found at $d h n 2, d h n 7, a b a R$ and chcs. On the basis of the average proportion of nearestneighbor haplotypes that are present in the same locality $\left(S_{\mathrm{nn}}\right)$ both groups were differentiated at $d h n 2$, dhn7, dhy2PP and a3iP2 $(P<0.001-0.05)$. Two loci, dhn2 and $d h n 7$, also showed high similarity between pairs of sequences derived from each region $\left(K_{\mathrm{ST}}=0.098\right.$ and 0.067 , respectively, $P<0.01$ ).

Significant differentiation was found between Scottish populations vs continental European populations measured as an average of $F_{\mathrm{ST}}$ values over all polymorphic sites detected (Table 4). The only exceptions include southern Scottish populations as compared with northern and central Europe, eastern Scottish compared with northern European and western Scottish compared with Spanish populations.

Analysis of genetic clustering with full sequence data gave the best support for all individuals from European mainland and Scottish populations at eight loci and for individuals from Scottish populations at 12 loci belonging to one genetic cluster. At all polymorphic sites and indels at both eight and 12 loci, the best support was obtained for four clusters, but without clear pattern of geographical distribution (Supplementary Figure S3).

\section{Coalescent simulations}

For each geographic group of Scottish Scots pine populations the observed pattern of the frequency distribution spectrum was not compatible with either the standard neutral or growth models. In simulations under the standard neutral model and growth model the mean Tajima's $D$ was significantly lower and Fay and Wu's $H$ significantly higher than the observed values except for the southern group, the only one with positive mean $H$ values (Table 5, Supplementary Table S8). Among the 20 different bottleneck models tested the most compatible for the western group was a relatively recent bottleneck $(t=0.00125)$ that reduced the population to $2 \%$ of the present size followed by moderate population growth (Table 5, Supplementary Table S9). This model also held for the eastern group, but was always rejected for the southern group, where different bottleneck scenarios never led to positive values for both Tajima's $D$ and Fay and Wu's $H$ statistics (Supplementary Table S9). In general, the simulations indicate heterogeneity in the allelic frequency distribution among geographical regions in Scotland.

\section{Discussion}

\section{Multi-locus signatures of population history}

The Scottish populations showed clear molecular signatures of different demographic histories. Across all regions, the allele frequency distribution was skewed towards intermediate frequency polymorphisms, and the rate of decline of $\mathrm{LD}$ was reduced and nucleotide diversity levels were equivalent to or higher than continental European populations of the species. The skew of allelic frequency distribution, apparent as positive values of Tajima's $D$, was in clear contrast to previous reports for this species in continental Europe (Palmé et al., 2008; Wachowiak et al., 2009) and for published studies of other species (North American Douglas fir, Eckert et al., 2009; P. taeda González-Martínez et al., 2006a; other conifer species Savolainen and Pyhäjärvi, 2007; European Quercus petraea Derory et al., 
2009; Populus tremula Ingvarsson, 2005), where negative values of Tajima's $D$ have been found. In these species, the excess of low frequency-derived mutations has been ascribed to the influence of postglacial range expansion (Brown et al., 2004; Pyhäjärvi et al., 2007) or potentially the influence of recurrent selective sweeps (for example, Eckert et al., 2009). In contrast, rather than range expansion, the bias towards intermediate-frequency polymorphisms in Scottish populations suggests the influence of a bottleneck although, as shown in recent simulation studies, a skew of allelic frequency variants may also result from pooling local samples with different demographic histories (Städler et al., 2009). However, the bottleneck hypothesis was also supported by the overall pattern of LD, which showed a reduced rate of decline relative to continental European populations of the species. In coalescent simulations, the bottleneck scenario fits best for western populations and the data were compatible with a relatively recent, severe bottleneck. Depending on the effective population size and generation time assumed, this bottleneck ended a maximum of a few tens of thousands of ya (for example, about 25000 ya assuming $\left.N_{\mathrm{e}}=200000\right)$. Bottlenecking is expected to increase association (correlation among sites with distance) of alleles and polymorphic sites across loci. In Scottish populations, the decay of LD was almost three times slower than that in mainland populations. Reduced decay of LD has also been observed in populations of American P. taeda that had probably experienced bottlenecks (Brown et al., 2004; González-Martínez et al., 2006a) and contrasting allele frequency distributions were observed between northern populations and recently bottlenecked southern populations of Quercus crispula in Japan (where the latter showed positive Tajima's D, Quang et al., 2008).

Although there are exceptions (Grivet et al., 2009), it is expected that bottlenecks should have a stronger impact on the allele frequency distribution spectrum and LD than on the overall level of diversity (Wright et al., 2005). Long-lived, wind-pollinated tree species should be capable of maintaining genetic diversity even during range shifts; that is, they are buffered against rapid changes in genetic variation due to fluctuations in population size (Austerlitz et al., 2000). Indeed, relative to mainland European populations of Scots pine, Scottish populations did not show a decline in nucleotide diversity, as is expected where colonization has been relatively recent (Nei et al., 1975; Pannell and Dorken, 2006). In fact, genetic variation in Scottish populations seems to be slightly higher than in mainland populations $\left(\theta_{\text {sil }}=0.011\right.$ vs 0.009 , respectively) and relative to previous estimates for the species $\left(\theta_{\text {sil }}=0.005\right.$ at 16 loci with some related to timing of bud set (Pyhäjärvi et al., 2007) and $\theta_{\text {sil }}=0.0089$ at 14 cold-tolerance candidate loci (Wachowiak et al., 2009)). Compared with estimates in other forest tree species, overall diversity in Scottish populations $\left(\pi_{\mathrm{tot}}=0.0078\right)$ is only lower than that in broadleaved Populus tremula (0.0111, Ingvarsson, 2005) and is higher than that in Q. crispula, (0.0069, Quang et al., 2008), Q. petraea (0.0062, Derory et al., 2009), $P$. pinaster (0.0055, Eveno et al., 2008), P. taeda (0.0040, Brown et al., 2004), Picea abies (0.0039, Heuertz et al., 2006) and other conifers (Savolainen and Pyhäjärvi, 2007). The diversity estimate for Scottish populations is compatible with the patterns of genetic variation observed in previous studies (monoterpenes Forrest, 1980; Forrest, 1982), allozymes Kinloch et al., 1986), chloroplast DNA microsatellite markers Provan et al., 1998).

Although it seems clear that bottlenecking has been an influence on Scottish populations, estimation of the timing of the event is heavily dependent on various assumptions, including the effective population size and generation time estimates. For instance, in continental populations of Norway spruce and Scots pine, simulation studies suggested a rather ancient bottleneck that ended several hundred thousand to more than one million ya, respectively (Lascoux et al., 2008). In our data, coalescent simulation of various demographic scenarios supported the conclusion that bottlenecking had occurred, but suggested more recent timing. A similar signal, suggesting bottlenecking on a timescale related to the most recent glaciation, was detected in Italian populations of Aleppo pine (Grivet et al., 2009). Furthermore, the severity of the bottleneck experienced by Scottish populations appears to have been strong enough to account for the observed discrepancy in allelic frequency distributions and decay of LD, in contrast to continental European tree populations (Lascoux et al., 2008).

However, as we observed heterogeneity in the pattern of nucleotide diversity among regions within Scotland, it seems likely that different parts of the population have experienced different demographic histories. The ratio of recombination to diversity and the level of LD in western Scottish populations were similar to those in mainland European populations, but about three times higher than those in southern and eastern Scottish groups. Various bottleneck scenarios could be clearly rejected for the southern group in our coalescent simulation analysis. The homogenizing effects of gene flow on genetic diversity are well known for highly outcrossing windpollinated species, and there is evidence for historically high gene flow among Scottish populations from work using chloroplast markers (Provan et al., 1998). In addition, molecular and isozyme studies provide no suggestion of a difference in outcrossing rates between regions that could account for a difference in spatial distribution of polymorphism (Kinloch et al., 1986). As, until recently, Scots pine covered large parts of Scotland, differentiation between regional groups due to genetic drift also seems unlikely, as this should be most significant for small populations (Pannell and Dorken, 2006). Inter-regional differences also seem unlikely to be the result of selection. If this was the case, we would expect differences in the frequency distribution spectrum between groups or at least reduced diversity levels at selected loci. However, the observed dominance of intermediate frequency variants in all groups together with very rapid decay of LD (within a few hundred base pairs) excludes a selective sweep as an explanation. Furthermore, nucleotide and haplotype diversity is at least as high in southern and eastern groups as in the western group, whereas directional selection should reduce diversity. Therefore, overall, historical changes in population size and distribution seem a more plausible explanation for the pattern of nucleotide variation in Scottish populations and, as a single migration and bottleneck event cannot account for the observed pattern of diversity, it seems that heterogeneity within the Scottish population is most likely to be the result of admixture of populations from different origins. 
Compared with continental Europe, southern and eastern groups of Scottish pines showed no overall difference in allele frequency distribution at polymorphic sites from north or central European populations, but differentiation from Spanish and Turkish populations. On the other hand, the western group was significantly differentiated from all mainland populations except those from Spain. In previous studies, populations from the west of Scotland were more closely related to southern European populations in monoterpene composition and isozyme frequency (Forrest, 1982) or geographically structured $m t$ DNA variation (Sinclair et al., 1998) than to populations from north-central Europe, which were more similar to the southern and eastern Scottish pinewoods. Similarities between western Scotland and south European Scots pine could simply be stochastic, due to homogenising selection for similar environments or, alternatively, could reflect common ancestry of the populations. Genetic similarity at mtDNA markers (maternally transmitted in pines) suggests the latter. However, as Iberian populations did not contribute to the most recent recolonization of central and northern Europe (Tobolski and Hanover, 1971; Prus-Glowacki and Stephan, 1994; Soranzo et al., 2000; Pyhäjärvi et al., 2008), this genetic similarity would reflect a common origin predating the last glacial period. Therefore, contemporary Scottish populations may originate from western populations that survived the last glaciation in southwestern parts of the British Isles, western continental Europe (Ballantyne and Harris, 1994; Bennett, 1995) or now-submerged parts of the continental shelf. Future genetic studies at more loci (including new $m t$ DNA markers) and in more populations would allow more precise assessment of the spatial distribution of haplotypes in Scottish and mainland populations and testing of colonization hypotheses. This should soon be feasible as new genomic resources for pine, including multiple nuclear and $m t$ DNA loci, are currently being developed (for example, through the EVOLTREE Network of Excellence).

\section{Effects of selection at individual loci}

At mutation-drift equilibrium, genetic drift and gene flow influence the level of differentiation between populations for selectively neutral markers (Kawecki and Ebert, 2004; Savolainen et al., 2007). Little differentiation between Scottish and mainland European populations of Scots pine at neutral markers (Kinloch et al., 1986; Prus-Glowacki and Stephan, 1994; Provan et al., 1998), but divergence at quantitative traits for characters of adaptive importance (for example, phenology, growth and survival rates, Ennos et al., 1998; Worrell, 1992; Hurme et al., 1997) suggests that selection is driving adaptive differentiation in both geographical regions. As they differ significantly in climatic, edaphic and biotic conditions, it is possible that observed nucleotide and/or haplotype differentiation at dhn2 and $d h n 7$ and some differences in the allele-frequency spectrum at dhy2PP, abaR, a3iP2 and chcs may be due to selection. Similarly, reduced nucleotide and haplotype diversity and a difference in the frequency and distribution of polymorphism found at $1 p 3-1$ and ccoaomt in the western as compared with the southern and eastern groups of Scottish populations could have been affected by diversifying selection at the range edge where populations are under direct oceanic influence. In contrast, the haplotype dimorphism at lp3-3 could potentially result from the long-term action of balancing selection, maintaining variation across geographical regions. However, as admixture at $l p 3-3$ cannot be ruled out, a study of nucleotide polymorphism in mainland European populations would be necessary to verify whether or not balancing selection has been an influence at this locus.

Some of the loci analyzed showed distinct nucleotide diversity patterns relative to genetic background in other species (for example, lp3-1 and ccoaomt in P. pinaster Eveno et al., 2008, ccoaomt in P. taeda, González-Martínez et al., 2006a). Although there is accumulating evidence on the polygenic character of adaptive traits from quantitative trait loci studies (Howe et al., 2003; Buckler et al., 2009), it remains unclear whether or not there are genes of major effect that contribute to adaptive variation in conifers. In the case of Scottish pinewoods, adaptation was probably driven by postglacial migration from a predominantly continental to an oceanic environment over the past $\sim 7000$ yrs. For long-lived conifers, adaptive differentiation would be expected to occur over several dozens of generations after vicariance. However, even though selection can be very effective in species with large population sizes, the time since the last glaciation seems too short for pine species to have accumulated new mutations that could be rapidly fixed by selection. Adaptive divergence is, therefore, more likely to result from selection acting on standing variation, which may have arisen in endemic populations that survived last glaciations in Western Europe or the British Isles. Moreover, as differentiation at the trait level in forest trees is likely to result from allelic associations among large numbers of loci, rather than changes in allelic frequencies at individual loci, the signature of selection may be more readily detectable as covariance of allele frequencies at multiple loci (Le Corre and Kremer, 2003; Latta, 2004; Derory et al., 2009). Therefore many more loci, including regulatory regions (to date, generally omitted from analyses of nucleotide variation in conifers), would need to be studied in parallel before the influence of selection could be verified. Scottish populations, which show considerable ecological, phenotypic and genetic diversity over short geographic distances, represent an excellent study system for multi-locus analysis of complex trait variation (Neale and Savolainen, 2004; González-Martínez et al., 2006b). Such studies will, however, have to take into account the potential role of recent population history in shaping patterns of nucleotide diversity, and, therefore, ensure that sampling is conducted at sufficient density to control for historical influences. Association studies of allelic variants and adaptive variation at quantitative traits between individuals from different, locally adapted populations could also better validate the signatures of selection and the functional role of the nuclear genes studied.

\section{Conflict of interest}

The authors declare no conflict of interest. 


\section{Acknowledgements}

WW acknowledges financial support from the Polish Ministry of Science (grant nr 3653/B/P01/2008/35), NERC and EU Network of Excellence EVOLTREE (mobility grant). MJS is a PhD student supported by the Scottish Forestry Trust. We thank Dave Sim, Joan Beaton and Ben Moore (Macaulay Institute) for making the seed collections, the owners of the woodlands for their cooperation and Joan Cottrell (Forest Research) and anonymous reviewers for constructive comments on the paper.

\section{References}

Achaz G (2009). Frequency Spectrum Neutrality Tests: One for All and All for One. Genetics 183: 249-258.

Ahuja MR, Neale DB (2005). Evolution of genome size in conifers. Silvae Genet 54: 126-137.

Austerlitz F, Mariette S, Machon N, Gouyon PH, Godelle B (2000). Effects of colonization processes on genetic diversity: Differences between annual plants and tree species. Genetics 154: 1309-1321.

Ballantyne CK, Harris C (1994). The Periglaciation of Great Britain. Cambridge University Press: Cambridge, 330pp.

Bennett KD (1995). Post-glacial dynamics of pine (Pinus sylvestris) and pinewoods in Scotland. In: Aldhous JR (ed). Scottish Natural Heritage. Forestry Commission, The Royal Society for the Protection of Birds: Edinburgh. pp 23-39.

Birks HJB (1989). Holocene isochrone maps and patterns of tree-spreading in the British Isles. Journal of Biogeography 16: 503-540.

Brown GR, Gill GP, Kuntz RJ, Langley CH, Neale DB (2004). Nucleotide diversity and linkage disequilibrium in loblolly pine. Proc Natl Acad Sci U S A 101: 15255-15260.

Buckler ES, Holland JB, Bradbury PJ, Acharya CB, Brown PJ, Browne C et al. (2009). The Genetic Architecture of Maize Flowering Time. Science 325: 714-718.

Cheddadi R, Vendramin G, Litt T, François L, Kageyama M, Lorentz $S$ et al. (2006). Imprints of glacial refugia in the modern genetic diversity of Pinus sylvestris. Glob Ecol Biogeogr 15: $271-282$.

Corander J, Tang J (2007). Bayesian analysis of population structure based on linked molecular information. Math Biosci 205: 19-31.

Critchfield WB, Little E (1965). US Department of Agriculture, pp 97.

Derory J, Scotti-Saintagne C, Bertocchi E, Le Dantec L, Graignic $\mathrm{N}$, Jauffres A et al. (2009). Contrasting relationships between the diversity of candidate genes and variation of bud burst in natural and segregating populations of European oaks. Heredity 104: 438-448.

Dvornyk V, Sirvio A, Mikkonen M, Savolainen O (2002). Low nucleotide diversity at the pal1 locus in the widely distributed Pinus sylvestris. Mol Biol Evol 19: 179-188.

Eckert AJ, Wegrzyn JL, Pande B, Jermstad KD, Lee JM, Liechty JD et al. (2009). Multilocus Patterns of Nucleotide Diversity and Divergence Reveal Positive Selection at Candidate Genes Related to Cold Hardiness in Coastal Douglas Fir (Pseudotsuga menziesii var. menziesii. Genetics 183: 289-298.

Ennos RA, Worrell R, Malcolm DC (1998). The genetic management of native species in Scotland. Forestry 71: 1-23.

Eveno E, Collada C, Guevara MA, Leger V, Soto A, Diaz L et al. (2008). Contrasting patterns of selection at Pinus pinaster Ait. drought stress candidate genes as revealed by genetic differentiation analyses. Mol Biol Evol 25: 417-437.

Excoffier L, Laval G, Schneider S (2005). Arlequin ver. 3.0: An integrated software package for population genetics data analysis. Evolutionary Bioinformatics Online 1: 47-50.
Fay JC, Wu C-I (2000). Hitchhiking under positive darwinian selection. Genetics 155: 1405-1413.

Forrest GI (1980). Genotypic variation among native Scots pine populations in Scotland based on monoterpene analysis. Forestry 53: 101-128.

Forrest GI (1982). Relationship of some European Scots pine populations to native Scottish woodlands based on monoterpene analyses. Forestry 55: 19-37.

García-Gil MR, Mikkonen M, Savolainen O (2003). Nucleotide diversity at two phytochrome loci along a latitudinal cline in Pinus sylvestris. Mol Ecol 12: 1195-1206.

González-Martínez SC, Ersoz E, Brown GR, Wheeler NC, Neale DB (2006a). DNA sequence variation and selection of tag single-nucleotide polymorphisms at candidate genes for drought-stress response in Pinus taeda L. Genetics 172: 1915-1926.

González-Martínez SC, Krutovsky KV, Neale DB (2006b). Forest-tree population genomics and adaptive evolution. New Phytologist 170: 227-238.

Grivet D, Sebastiani F, González-Martínez SC, Vendramin GG (2009). Patterns of polymorphism resulting from long-range colonization in the Mediterranean conifer Aleppo pine. New Phytologist 184: 1016-1028.

Haddrill PR, Thornton KR, Charlesworth $\mathrm{B}$, Andolfatto $\mathrm{P}$ (2005). Multilocus patterns of nucleotide variability and the demographic and selection history of Drosophila melanogaster populations. Genome Res 15: 790-799.

Hamrick JL, Godt MJW, Sherman-Broyles SL (1992). Factors influencing levels of genetic diversity in woody plants species. New For 6: 95-124.

Heuertz M, De Paoli E, Kallman T, Larsson H, Jurman I, Morgante $M$ et al. (2006). Multilocus Patterns of Nucleotide Diversity, Linkage Disequilibrium and Demographic History of Norway Spruce [Picea abies (L.) Karst]. Genetics 174: 2095-2105.

Hill WG, Robertson A (1968). Linkage disequilibrium in finite populations. Theor Appl Genet 38: 226-231.

Howe GT, Aitken SN, Neale DB, Jermstad KD, Wheeler NC, Chen THH (2003). From genotype to phenotype: unraveling the complexities of cold adaptation in forest trees. Canadian Journal of Botany-Revue Canadienne De Botanique 81: 1247-1266.

Hudson RR (2000). A New Statistic for Detecting Genetic Differentiation. Genetics 155: 2011-2014.

Hudson RR (2002). Generating samples under a Wright-Fisher neutral model of genetic variation. Bioinformatics 18: 337-338.

Hudson RR, Boos DD, Kaplan NL (1992). A statistical test for detecting geographic subdivision. Mol Biol Evol 9: 138-151.

Hudson RR, Kreitman M, Aguade M (1987). A Test of Neutral Molecular Evolution Based on Nucleotide Data. Genetics 116: 153-159.

Hughes AL, Nei M (1988). Pattern of nucleotide substitution at major histocompatibility complex class I loci reveals overdominant selection. Nature 335: 167-170.

Huntley B, Birks HJB (1983). An Atlas of Past and Present Pollen Maps for Europe: 0-13000 Years Ago. Cambridge University Press: Cambridge. 667pp.

Hurme P, Repo T, Savolainen O, Paakkonen T (1997). Climatic adaptation of bud set and frost hardiness in Scots pine (Pinus sylvestris). Can J For 27: 716-723.

Ingvarsson PK (2005). Nucleotide polymorphism and linkage disequilbrium within and among natural populations of European Aspen (Populus tremula L. Salicaceae) Genetics 169: 945-953.

Joosen RVL, Lammers M, Balk PA, Bronnum P, Konings MCJM, Perks $\mathrm{M}$ et al. (2006). Correlating gene expression to physiological parameters and environmental conditions during cold acclimation of Pinus sylvestris, identification of molecular markers using cDNA microarrays. Tree Physiol 26: 1297-1313.

Karhu A, Hurme P, Karjalainen M, Karvonen P, Kärkkäinen K, Neale D et al. (1996). Do molecular markers reflect patterns of differentiation in adaptive traits of conifers? Theor Appl Genet 93: 215-221. 
Kawecki TJ, Ebert D (2004). Conceptual issues in local adaptation. Ecology Letters 7: 1225-1241.

Kinloch BB, Westfall RD, Forrest GI (1986). Caledonian Scots pine origins and genetic structure. New Phytologist 104: 703-729.

Lascoux M, Pyhäjärvi T, Källman T, Savolainen O (2008). Past demography in forest trees: what can we learn from nuclear DNA sequences that we do not already know? Plant Ecology $\mathcal{E}$ Diversity 1: 209-215.

Latta RG (2004). Relating processes to patterns of genetic variation across landscapes. Forest Ecology and Management 197: 91-102.

Le Corre V, Kremer A (2003). Genetic variability at neutral markers, quantitative trait loci and trait in a subdivided population under selection. Genetics 164: 1205-1219.

Librado P, Rozas J (2009). DnaSP v5: A software for comprehensive analysis of DNA polymorphism data. Bioinformatics 25: 1451-1452.

Lynch M, Crease TJ (1990). The analysis of population survey data on DNA sequence variation. Mol Biol Evol 7: 377-394.

Mason WL, Hampson A, Edwards C (2004). Managing the Pinewoods of Scotland. Forestry Commission: Edinburgh.

McDonald JH, Kreitman M (1991). Adaptive protein evolution at the Adh locus in Drosophila. Nature 351: 652-654.

Neale DB, Ingvarsson PK (2008). Population, quantitative and comparative genomics of adaptation in forest trees. Curr Opin Plant Biol 11: 149-155.

Neale DB, Savolainen O (2004). Association genetics of complex traits in conifers. Trends Plant Sci 9: 325-330.

Nei M (1987). Molecular Evolutionary Genetics. Columbia University Press: New York.

Nei M, Maruyama T, Chakraborty R (1975). The Bottleneck Effect and Genetic Variability in Populations. Evolution 29: 1-10.

Palmé AE, Wright M, Savolainen O (2008). Patterns of divergence among conifer ESTs and polymorphism in Pinus sylvestris identify putative selective sweeps. Mol Biol Evol 25: 2567-2577.

Pannell J, Dorken M (2006). Colonisation as a common denominator in plant metapopulations and range expansions: effects on genetic diversity and sexual systems. Landscape Ecology 21: 837-848.

Perks MP, Ennos RA (1999). Analysis of genetic variation for quantitative characters between and within four native populations of Scots pine (Pinus sylvestris). Botanical Journal of Scotland 51: 103-110.

Perks MP, McKay HM (1997). Morphological and physiological differences in Scots pine seedlings of six seed origins. Forestry 70: 223-232.

Petit RJ, Aguinagalde I, de Beaulieu JL, Bittkau C, Brewer S, Cheddadi R et al. (2003). Glacial refugia: Hotspots but not melting pots of genetic diversity. Science 300: 1563-1565.

Provan J, Soranzo N, Wilson NJ, McNicol JW, Forrest GI, Cottrell J et al. (1998). Gene-pool variation in Caledonian and European Scots pine (Pinus sylvestris L.) revealed by chloroplast simple-sequence repeats. Proceedings of the Royal Society of London Series B-Biological Sciences 265: 1697-1705.

Prus-Glowacki W, Stephan BR (1994). Genetic variation of Pinus sylvestris from Spain in relation to other European populations. Silvae Genet 43: 7-14.

Pyhäjärvi T, Garcia-Gil MR, Knürr T, Mikkonen M, Wachowiak W, Savolainen O (2007). Demographic History Has Influ- enced Nucleotide Diversity in European Pinus sylvestris Populations. Genetics 177: 1713-1724.

Pyhäjärvi T, Salmela MJ, Savolainen O (2008). Colonization routes of Pinus sylvestris inferred from distribution of mitochondrial DNA variation. Tree Genetics \& Genomes 4: 247-254.

Quang ND, Ikeda S, Harada K (2008). Nucleotide variation in Quercus crispula Blume. Heredity 101: 166-174.

Savolainen O, Pyhäjärvi T (2007). Genomic diversity in forest trees. Curr Opin Plant Biol 10: 162-167.

Savolainen O, Pyhäjärvi T, Knürr T (2007). Gene flow and local adaptation in trees. Annual Review of Ecology, Evolution, and Systematics 38: 595-619.

Sinclair WT, Morman JD, Ennos RA (1998). Multiple origins for Scots pine (Pinus sylvestris L.) in Scotland: evidence from mitochondrial DNA variation. Heredity 80: 233-240.

Soranzo N, Alia R, Provan J, Powell W (2000). Patterns of variation at a mitochondrial sequence-tagged-site locus provides new insights into the postglacial history of European Pinus sylvestris populations. Molecular Ecology 9: 1205-1211.

Stadler T, Haubold B, Merino C et al. (2009). The impact of sampling schemes on the site frequency spectrum in nonequilibrium subdivided populations. Genetics 182: 205-216.

Steven HM, Carlisle A (1959). The Native Pinewoods of Scotland. Oliver and Boyd: Edinburgh.

Svendsen JI, Astakhov VI, Bolshiyanov DY, Demidov I, Dowdeswell JA, Gataullin V et al. (1999). Maximum extent of the Eurasian ice sheets in the Barents and Kara Sea region during the Weichselian. Boreas 28: 234 - 242.

Tajima F (1989). Statistical-method for testing the neutral mutation hypothesis by DNA polymorphism. Genetics $\mathbf{1 2 3}$ 585-595.

Thornton K (2005). Recombination and the properties of Tajima's D in the context of approximate likelihood calculation. Genetics 171: 2143-2148.

Tobolski JJ, Hanover JW (1971). Genetic Variation in the Monoterpenes of Scotch pine. Forest Science 17: 293-299.

Wachowiak W, Balk P, Savolainen O (2009). Search for nucleotide diversity patterns of local adaptation in dehydrins and other cold-related candidate genes in Scots pine (Pinus sylvestris L.). Tree Genetics \& Genomes 5: 117-132.

Wang XR, Szmidt AE, Lindgren D (1991). Allozyme differentiation among populations of Pinus sylvestris L. FROM Sweden and China. Hereditas 114: 219-226.

Watterson GA (1975). On the number of segregating sites in genetical models without recombination. Theor Popul Biol 7: 256-276.

Willis KJ, van Andel TH (2004). Trees or no trees? The environments of central and eastern Europe during the Last Glaciation. Quat Sci Rev 23: 2369-2387.

Worrell R (1992). A Comparison Between European Continental and British Provenances of Some British Native Trees: Growth, Survival and Stem Form. Forestry 65: 253-280.

Wright SI, Bi IV, Schroeder SG, Yamasaki M, Doebley JF, McMullen MD et al. (2005). The Effects of Artificial Selection on the Maize Genome. Science 308: 1310-1314.

Wright SI, Gaut BS (2005). Molecular population genetics and the search for adaptive evolution in plants. Mol Biol Evol 22: 506-519.

Supplementary Information accompanies the paper on Heredity website (http://www.nature.com/hdy) 